\title{
Correction to: Overexpression of miR-124 Protects Against Neurological Dysfunction Induced by Neonatal Hypoxic-Ischemic Brain Injury
}

\author{
Liulin Xiong ${ }^{1,2}$ - Haoli Zhou ${ }^{3} \cdot$ Qiong Zhao ${ }^{4} \cdot$ Lulu Xue $^{3} \cdot$ Mohammed Al-Hawwas $^{2}$. Jingyuan $\mathrm{He}^{5} \cdot$ Maxiu Wu $^{3}$. \\ Yu Zou ${ }^{3} \cdot$ Mingan Yang ${ }^{6} \cdot$ Jing Dai ${ }^{3} \cdot$ Manxi He ${ }^{3} \cdot$ Tinghua Wang ${ }^{1,3,5}$
}

Published online: 28 December 2021

(c) Springer Science+Business Media, LLC, part of Springer Nature 2021

\section{Correction to:}

Cellular and Molecular Neurobiology (2020) 40:737-750 https://doi.org/10.1007/s10571-019-00769-2

The original version of this article unfortunately contained incorrect part figures of Figs. 1c and 3a.

Hence, the correct figures of Figs. 1 and 3 with its caption is presented here.

The original article has been corrected.

Publisher's Note Springer Nature remains neutral with regard to jurisdictional claims in published maps and institutional affiliations.

Liulin Xiong and Haoli Zhou are Co-first authors.

Liulin Xiong, Haoli Zhou, Manxi He, and Tinghua Wang have contributed equally to this work.

The original article can be found online at https://doi.org/10.1007/ s10571-019-00769-2.

Manxi He

2357622298@qq.com

$\triangle$ Tinghua Wang

tinghua_neuron@263.net

1 Department of Anesthesiology, The Affiliated Hospital of Zunyi Medical University, Zunyi 563000, Guizhou, China

2 School of Pharmacy and Medical Sciences, Faculty of Health Sciences, University of South Australia, Adelaide, SA 5000, Australia

3 The Clinical Hospital of Chengdu Brain Science Institute, MOE Key Lab for Neuroinformation, University of Electronic Science and Technology of China, Chengdu 611731, China
4 Department of Anesthesiology, Sun Yat-Sen Memorial Hospital, Sun Yat-Sen University, Guangzhou 510120, Guangdong, China

5 Institute of Neuroscience, Animal Zoology Department, Kunming Medical University, Kunming 650031, China

6 Division of Biostatistics and Epidemiology, School of Public Health, San Diego State University, San Diego 92182, USA 
Fig. 1 Successful establishment of HI and OGD models. a The line chart of the zea-longa score at $0 \mathrm{~h}, 6 \mathrm{~h}, 12 \mathrm{~h}, 24 \mathrm{~h}$ and 48 $\mathrm{h}$ after $\mathrm{HI}$ in the sham and $\mathrm{HI}$ groups. Data are analyzed using two-way repeated measures ANOVA and presented as the mean $\pm \mathrm{SD}(* * p<0.01)$. b MRI exhibited the cerebral infarction on the right side of the brain at 1 month after HI. The red arrow represents the infarct area. c Images showed the morphological changes of PC12, SHSY5Y and neurons under normal and OGD condition at $24 \mathrm{~h}$ after OGD, scale bar $50 \mu \mathrm{m}$

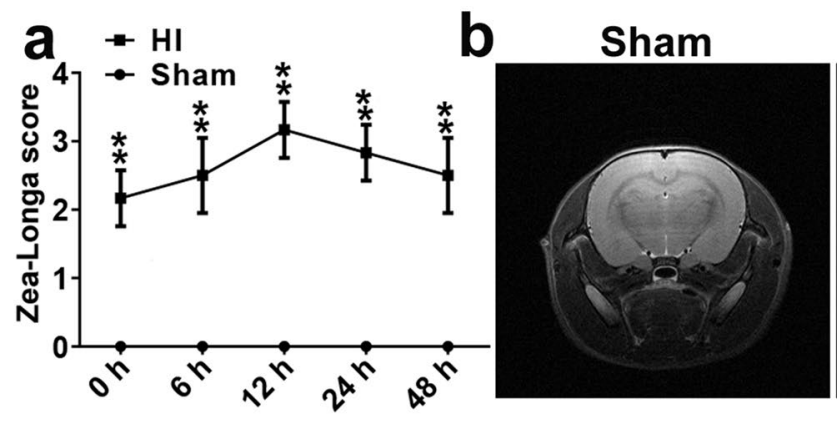

C

PC12
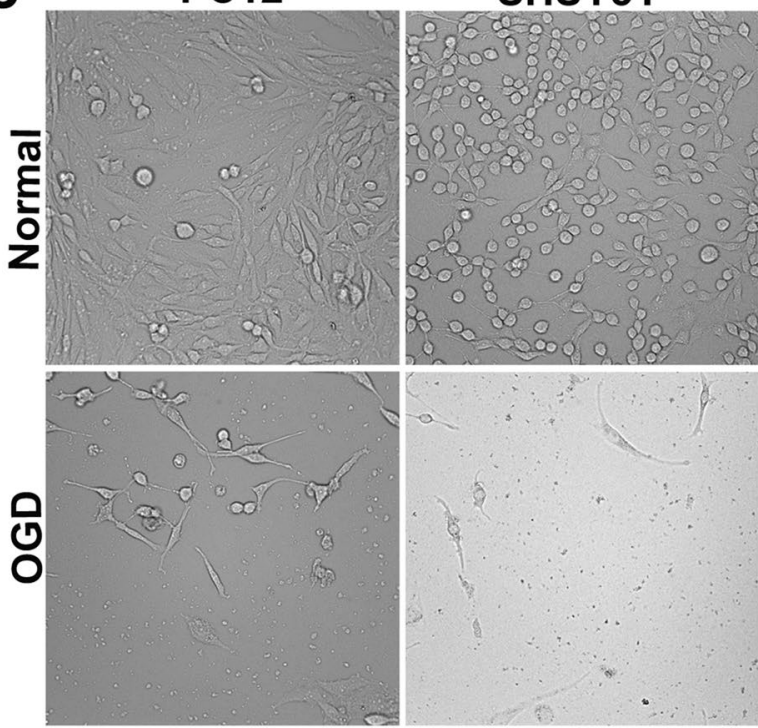

HI

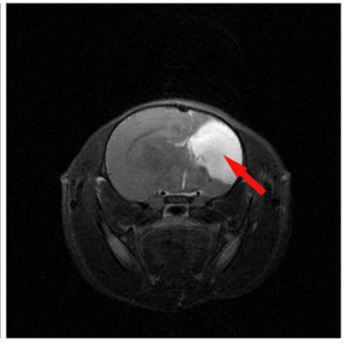

Neuron

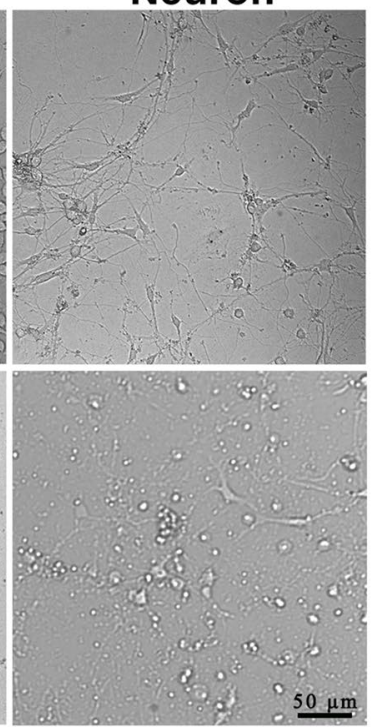


Fig. 3 The role of miR-124 overexpression in PC12, SHSY5Y, and neurons after OGD. a The images of morphological observation of PC12, SHSY5Y and neurons in bright-field condition among normal, OGD, OGD + miR-NC, and OGD + miR-124 groups. b The number of PC12 cells among normal, OGD, OGD + miRNC, and OGD + miR-124 groups at $24 \mathrm{~h}$ after OGD. c The number of SHSY5Y cells among normal, OGD, OGD + miR-NC, and OGD + miR-124 groups at $24 \mathrm{~h}$ post-OGD. d The number of survived neurons among normal, OGD, OGD + miR-NC, and OGD + miR-124 groups at $24 \mathrm{~h}$ post-OGD. Data are analyzed using one-way ANOVA and presented as the mean $\pm \mathrm{SD}(* * p<0.01)$. Scale bar $50 \mu \mathrm{m}$ a
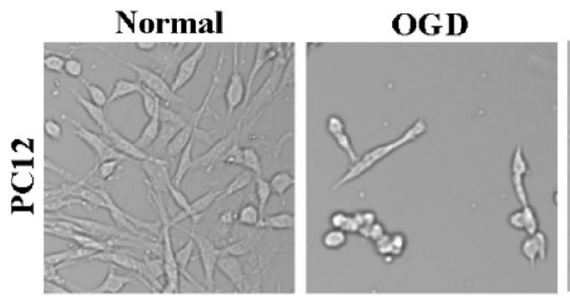

OGD+miR-NC

OGD+miR-124
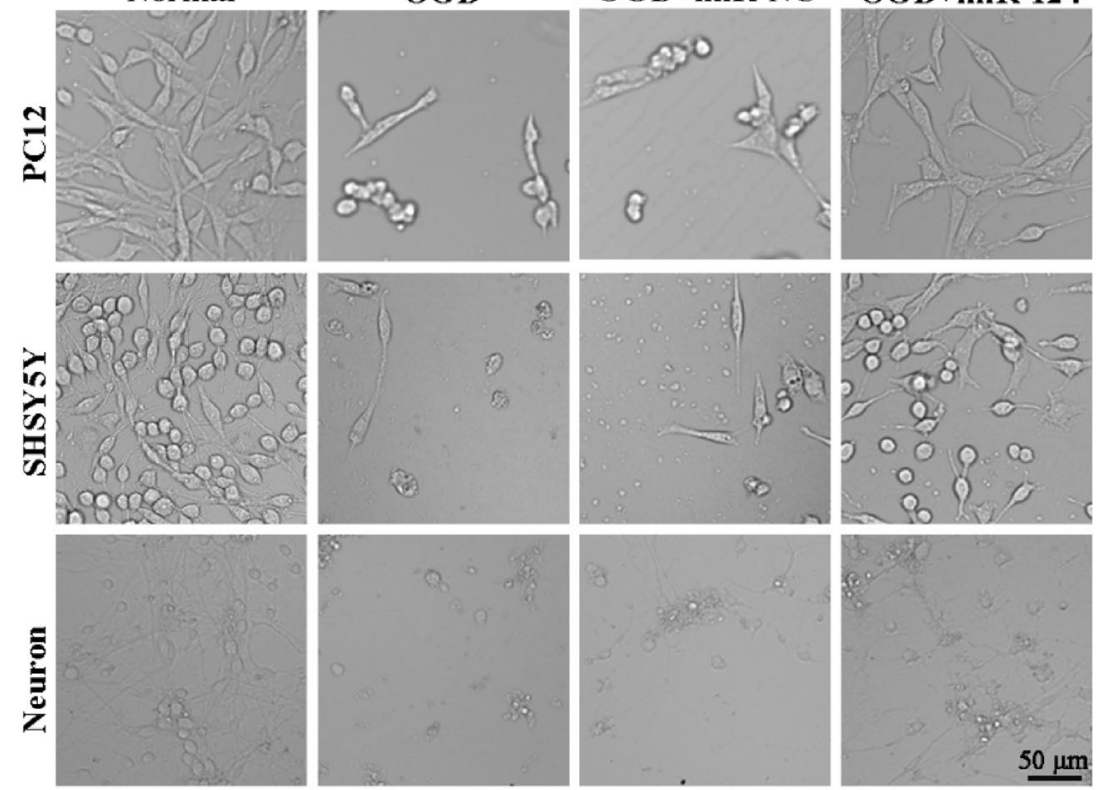

b
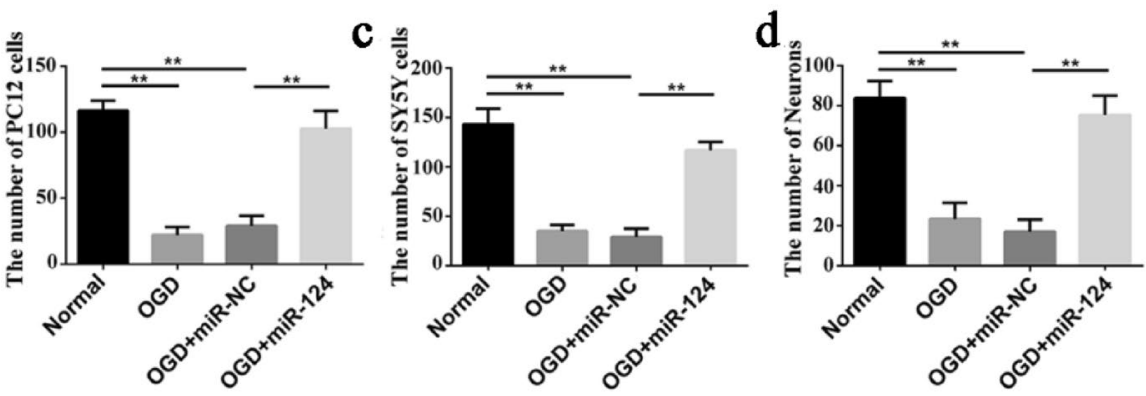